\title{
Deklinationsklassen. Zur vergleichenden Betrachtung der Substantivflexion ${ }^{1}$
}

\begin{abstract}
Deklinationsklassen bilden einen Grundpfeiler des traditionellen Paradigmenmodells, das nach dem Vorbild der Grammatiken der klassischen Sprachen auch für die Beschreibung der deutschen Substantivflexion Verwendung gefunden hat. Im vorliegenden Beitrag soll die Rolle, die Deklinationsklassen in der deutschen Substantivdeklination spielen, überprüft werden. Beobachtungen zur Substantivflexion in verschiedenen europäischen Sprachen, darunter das Ungarische, das Polnische und das Italienische, die unterschiedliche Positionen innerhalb eines Spektrums besetzen, das vom hochflexivischen Lateinischen bis zu Sprachen ohne Deklinationsklassen (wie dem Englischen oder dem Türkischen) reicht, liefern Bausteine für eine Neuanalyse der deutschen Substantivflexion. Sichtbar wird, dass die deutsche Substantivflexion, bildlich gesprochen, auf dem Weg vom „Typus Latein“ zum „Typus Englisch“ schon sehr viel weiter fortgeschritten ist, als dies traditionelle Darstellungen nahe legen. An die Stelle der für kanonische Deklinationsklassen charakteristischen Sätze von klassentypischen Flexiven ist eine kleine Menge von Mustern der Stammformenbildung getreten.
\end{abstract}

\section{Einleitung}

Im Deutschen besitzen die Lexeme der Wortarten Substantiv, Adjektiv, Pronomen und Verb jeweils eine Anzahl unterschiedlicher Flexionsformen, und daher stellt die Identifizierung, Analyse und Systematisierung der betreffenden Formen eine wesentliche Aufgabe der grammatischen Beschreibung dar. Diese Aufgabe kann auf unterschiedliche Art und Weise in Angriff genommen werden. Traditionell stehen aber im Mittelpunkt der Formenlehre die so genannten Deklinationen und Konjugationen. Im vorliegenden Beitrag soll die Rolle, die Deklinationsklassen in der deutschen Substantivflexion spielen, überprüft werden.

Deklinationsklassen sind zunächst einmal Teil eines morphologischen Modells, des Wort-und-Paradigma-Modells, das auf die klassischen Sprachen zugeschnitten ist, das aber zugleich die deutsche Grammatikschreibung seit ihren Anfängen mitgeprägt hat. Es orientiert sich an einem bestimmten Typ

Der vorliegende Aufsatz ist im Rahmen des Projekts „Grammatik des Deutschen im europäischen Vergleich“ (Leitung: Gisela Zifonun) am Institut für Deutsche Sprache (IDS), Mannheim, entstanden. Herzlichen Dank an Hardarik Blühdorn, Lutz Gunkel, Markus Hiller, Adriano Murelli, Renate Raffelsiefen, Susan Schlotthauer und Gisela Zifonun sowie an einen anonymen Gutachter für viele hilfreiche und weiterführende Hinweise! 
hochflexivischer Sprachen, für den das Lateinische das geläufigste Beispiel darstellt. Betrachtet man nur die indoeuropäischen Sprachen im heutigen Europa, so kann man demgegenüber das Englische als Beispiel einer Sprache ohne Deklinationsklassen nennen. Zwischen diesen Polen findet man eine vielfältige Abstufung von Sprachen, die unterschiedlich stark reduzierte Flexionssysteme besitzen. In den romanischen Sprachen ist die Kasusflexion fast ganz verschwunden, in den meisten slawischen Sprachen gut erhalten. Das Deutsche zeigt einen mittleren Grad von Flexionsabbau.

Dass mit dem Abbau von Komplexität in den Flexionssystemen auch die Rolle, die Deklinationsklassen zukommt, abnimmt, ist leicht beobachtbar, in den historischen Grammatiken beschrieben worden und entsprechend gut bekannt. Weniger klar ist oft, wo in dem so bezeichneten Spektrum einzelne moderne Sprachen ihren Platz haben. Insbesondere was das Deutsche angeht, herrscht erhebliche Unsicherheit bezüglich der Rolle, die Deklinationsklassen spielen oder nicht spielen.

Einer der Gründe liegt, wie ich im Folgenden deutlich machen möchte, darin, dass die deutsche Substantivflexion, bildlich gesprochen, auf dem Weg vom „Typus Latein“ zum „Typus Englisch“ schon sehr viel weiter fortgeschritten ist, als dies traditionelle Darstellungen nahe legen. Wenn dies zutrifft, sollte es nicht überraschen, dass die Anwendung von Beschreibungsmodellen, die eher auf den lateinischen Rand des Spektrums passen, beim Deutschen zu Schwierigkeiten führt. Symptomatisch ist die immer neu erörterte Frage nach der Anzahl und Einteilung der Deklinationsklassen im Deutschen. Verschiedene Gruppierungen und Taxonomien sind erwogen worden, aber ebenso wiederholt sich der Hinweis, dass eine anerkannte Systematik fehlt. Eisenberg fasst zusammen: „Grundsätzlich ist die Motiviertheit der Deklinationstypen aber bisher umstritten geblieben“ (Eisenberg 1998, S. 157). Es stellt sich die Frage: „Was tun mit Flexionsklassen?“ (Nübling 2008).

Ich möchte diese Frage hier über den Sprachvergleich angehen. Als Standardbeispiele für Sprachen mit entwickeltem bzw. fehlendem Deklinationsklassensystem können das Lateinische und das Türkische dienen (vgl. Plank 1991). Ich werde ferner das Ungarische, das Italienische und das Polnische heranziehen (in Abschnitt 2). Im vorliegenden Beitrag ist nur ein kurzer Blick auf die betreffenden Systeme möglich, aber auch so ergeben sich Hinweise, die für eine Analyse des Deutschen (in Abschnitt 3) genutzt werden können. ${ }^{2}$

Verwendete Abkürzungen: NOM (Nominativ), AKK (Akkusativ), DAT (Dativ), GEN (Genitiv), LOK (Lokativ), ABL (Ablativ), INS (Instrumental); M (Maskulinum), N (Neutrum), F (Femininum); SG (Singular), PL (Plural). Namen von Lexemen erscheinen in Versalien (z.B. HAUS), Namen von Wortformen in gewöhnlicher Orthographie (z.B. Häuser). 


\section{Deklinationsklassen im Sprachvergleich}

\subsection{Wozu Deklinationsklassen?}

Die lateinische Substantivflexion liefert das klassische Beispiel für ein System mit ausgeprägter Deklinationsklassenunterscheidung. Die Spezifika eines solchen Systems können durch den Vergleich mit Systemen ohne Deklinationsklasseneinteilung verdeutlicht werden. Im Falle des Englischen ist das Fehlen einer Deklinationsklasseneinteilung einfach eine Begleiterscheinung des weitgehenden Abbaus der Kasusflexion. Sprachen mit agglutinierender Flexion wie das Türkische liefern dagegen Beispiele für Systeme ohne Deklinationsklasseneinteilung, aber mit entwickeltem Kasussystem. Das Substantiv EV (,Haus') zeigt die folgenden Kasus- und Numerusflexive, die hier jeweils durch ein Paar aus Formangabe und Funktionsbestimmung dargestellt werden:

$$
-l e r / \mathrm{PL} \quad-i / \mathrm{AKK} \quad-e / \mathrm{DAT} \quad-i n / \mathrm{GEN} \quad-d e / \mathrm{LOK} \quad-d e n / \mathrm{ABL}
$$

Die bloße Grundform bildet den Nominativ Singular $(e v)$. Mittels der Suffixe können Formen wie evler, NOM.PL, evi, AKK.SG, oder evleri, AKK.PL, gebildet werden. Das Pluralsuffix erscheint gegebenenfalls in stammnäherer Position, ein Kasussuffix kann folgen. Die Reihenfolge entspricht allgemeinen Grundsätzen der Hierarchisierung nominaler Kategorien und verlangt keine besondere einzelsprachliche Festlegung.

In Abhängigkeit von der Lautform des Stamms können die Suffixe Varianten zeigen (insbesondere gemäß den Regeln der Vokalharmonie, ferner aufgrund von Stimmhaftigkeitsassimilation und zur Hiatus-Vermeidung). Solche Variantenbildungen sind automatisch und morphologisch irrelevant. Die Kasussuffixe gelten im Übrigen für alle Substantive und zwar, wie die Beispiele zeigen, ebenso für den Singular wie für den Plural. Form und Funktion stehen in einem Eins-zu-eins-Verhältnis. Die Beschreibung der Formenbildung der Substantive in einer türkischen Grammatik kann daher im Kern durch die bloße Aufzählung der Suffixe gegeben werden (vgl. Göksel/Kerslake 2005, S. 65, 67).

In Grammatiken des Lateinischen geschieht die Erfassung der Formenbildung traditionell durch die Aufstellung von Flexionsparadigmen. Die Paradigmen - wörtlich: Beispiele - präsentieren die Formen ausgewählter Lexeme und ordnen ihnen die relevanten morphosyntaktischen Kategorien zu, im Latein Numerus und Kasus, gewöhnlich in tabellarischer Form. Die so genannten Deklinationen fassen in der Regel mehrere Paradigmen zusammen, die systematisch aufeinander bezogen sind. Jede Deklination umgreift eine bestimmte, Version' der Formenbildung. Alle Substantive, deren Flexion mit den für eine Deklination gegebenen Musterbeispielen übereinstimmen, fallen in dieselbe Klasse. 
Für das klassische Latein werden traditionell fünf Deklinationen angenommen. Folgt man gängigen Annahmen zur Zerlegung der Formen in Stamm und Endung, so kann man (vereinfachend) fünf Sätze von Flexiven wie in Tabelle 1 ansetzen, wenngleich mit einem nicht unerheblichen Spielraum für Varianten innerhalb der Deklinationen (insbesondere in der 3. Deklination) gerechnet werden muss (Leumann 1977).

1. DEK. 2. DEK. 3. DEK. 4. DEK. 5. DEK.

\begin{tabular}{|c|c|c|c|c|c|}
\hline NOM.SG & $-a$ & $-u s$ & $-i s$ & $-u s$ & $-\bar{e} s$ \\
\hline VOK.SG & $-a$ & $-e$ & $-i s$ & $-u s$ & $-\bar{e} s$ \\
\hline AKK.SG & $-a m$ & $-u m$ & $-e m$ & $-u m$ & $-e m$ \\
\hline ABL.SG & $-\bar{a}$ & $-\overline{0}$ & $-e$ & $-\bar{u}$ & $-\bar{e}$ \\
\hline DAT.SG & $-a e$ & $-\bar{o}$ & $-\bar{\imath}$ & $-u \bar{\imath}$ & $-e i$ \\
\hline GEN.SG & $-a e$ & $-\bar{\imath}$ & $-i s$ & $-\bar{u} s$ & $-e i$ \\
\hline NOM.PL & $-a e$ & $-\bar{\imath}$ & $-\bar{e} s$ & $-\bar{u} s$ & $-\bar{e} s$ \\
\hline VOK.PL & $-a e$ & $-\bar{\imath}$ & $-\bar{e}$ & $-\bar{u} s$ & $-\bar{e} s$ \\
\hline AKK.PL & $-\overline{a s}$ & $-\overline{o s}$ & $-\bar{e} s$ & $-\bar{u} s$ & $-\bar{e} s$ \\
\hline ABL.PL & $-\bar{s}$ & $-\bar{s}$ & $-i b u s$ & $-i b u s$ & -èbus \\
\hline DAT.PL & $-\bar{s}$ & $-\bar{s}$ & $-i b u s$ & -ibus & -èbus \\
\hline GEN.PL & -ārum & -ōrum & $-u m$ & - uum & -èrum \\
\hline \multicolumn{6}{|l|}{ NEUTRUM } \\
\hline VOK/NOM/AKK.SG & - & $-u m$ & - & $-u$ & - \\
\hline VOK/NOM/AKK.PL & - & $-a$ & $-a$ & $-u a$ & - \\
\hline \multirow[t]{2}{*}{ Beispiellexeme: } & $\begin{array}{c}\text { CAPRA, F } \\
\text { (,Ziege) }\end{array}$ & $\begin{array}{c}\text { LUPUS, M } \\
\text { (,Wolf') }\end{array}$ & $\begin{array}{l}\text { CANIS, M } \\
\text { (,Hund') }\end{array}$ & $\begin{array}{l}\text { ICTUS, M } \\
\text { (,Schlag') }\end{array}$ & $\begin{array}{c}\text { RĒS, F } \\
\text { (,Sache) }\end{array}$ \\
\hline & & $\begin{array}{c}\text { IUGUM, N } \\
\text { (,Joch') }\end{array}$ & $\begin{array}{c}\text { NŌMEN, N } \\
\text { (,Name') }\end{array}$ & $\begin{array}{l}\text { GENU, N } \\
\text { (,Knie') }\end{array}$ & \\
\hline
\end{tabular}

Tab. 1: Latein: Flexivsätze

Lateinische Substantive werden zugleich in Genera (Kongruenzklassen) eingeteilt. Substantive der 1. und 5. Deklination sind meist Feminina, die der 2. und 4. Deklination dagegen Maskulina oder Neutra, während die 3. Deklination Substantive aller Genera umfasst. Neutra nehmen in den Kasus Ablativ, Dativ und Genitiv (mit kleinen Abweichungen) die gleichen Flexive wie die Maskulina. Die Neutra besitzen aber besondere Flexive für die Kasus Vokativ, Nominativ und Akkusativ, die im unteren Teil von Tabelle 1 angegeben sind. Im Neutrum fallen diese drei Kasus immer zusammen. 
Das Lateinische weicht stark von einer Eins-zu-eins-Beziehung von Form und Funktion ab. Numerus und Kasus werden nicht durch gesonderte Flexive markiert, sondern durch Endungen, die für Kasus-Numerus-Kombinationen stehen (z.B. -ärum für GEN.PL). Vor allem aber finden sich vielfach gleiche Endungen für unterschiedliche Funktionen, z.B., -ae, DAT/ GEN.SG und VOK/NOM.PL, 1. Deklination, und ebenso unterschiedliche Endungen für gleiche Funktionen, z.B. die fünf GEN.SG-Endungen (vgl. Wiese im Erscheinen). Dass für gleiche Funktionen in den verschiedenen Deklinationen je unterschiedliche Endungen verwendet werden, ist hier nicht etwa die Ausnahme, sondern ganz gewöhnlich. In einzelnen Positionen gibt es zwar Übereinstimmungen zwischen Paaren von Deklinationen wie z.B. im ABL/DAT.PL zwischen 1. und 2. Deklination (-is). Ferner finden sich Ähnlichkeiten zwischen Endungen verschiedener Deklination wie bei -ärum, -örum, -êrum, GEN.PL. Es gibt jedoch keine Endungen, die deklinationenübergreifend verwendet würden. Die Endungen der verschiedenen Deklinationen unterscheiden sich nicht nur hier und da, sondern bilden jeweils deklinationentypische Sätze, die sich als Ganze gegenüberstehen. Die lateinischen Deklinationen erfüllen damit in hohem Maße das Kriterium der Distinktivität, das Corbett (2009, S. 3, „distinctiveness") als definitorisch für ,kanonische ${ }^{6}$ Deklinationsklassen ansieht.

Wie die Behandlung der Neutra zeigt, müssen nicht alle Substantive, die zur gleichen Deklination gehören, bezüglich der Bildung aller Formen übereinstimmen. Wo Unterschiede in der Flexion der Substantive unabhängig vorhersagbar sind, setzt man in der lateinischen Grammatik keine Deklinationsklassen-Unterscheidungen an. Ein Neutrum wie IUGUM gehört wie das Maskulinum LUPUS zur 2. Deklination, obwohl die Flexion nur in einem Teil der Kasus übereinstimmt. Da der Unterschied durch das Genus bestimmt wird, wird keine zusätzliche Deklinationsklassenunterscheidung benötigt.

Die Einteilung in Deklinationsklassen hat also eine wohlbestimmte Aufgabe. Sie soll es, wenigstens im Prinzip, ermöglichen, die Flexionsformen beliebiger Substantive herzuleiten. Ist ein beliebiges Substantiv gegeben, so sollen sich seine Formen auf der Basis eines passenden Paradigmas durch geeignete Analogieschlüsse ermitteln lassen. Welche und wie viele Deklinationsklassen unterschieden werden, ergibt sich aus dieser Aufgabe. Nach der traditionellen Konzeption braucht man Deklinationsklassen überhaupt nur, weil - und nur insoweit - es Unterschiede in der Formenbildung gibt, für die keine anderen Gründe angeführt werden können als eben die Deklinationsklassenzugehörigkeit. Die lateinischen Deklinationen besitzen damit auch das zweite der beiden von Corbett für kanonische Deklinationsklassen geforderten Merkmale, die Unabhängigkeit (Corbett 2009, S. 5 f., ,independence ${ }^{\text {") }}$, d.h., die Verteilung der Lexeme auf die Deklinationsklassen ist nicht aufgrund ,externer' Eigenschaften (etwa phonologischer oder syntaktischer Eigenschaften) vorhersagbar. 
Der traditionelle Begriff der Deklination (oder Deklinationsklasse) unterscheidet sich von dem der (nominalen) Flexionsklasse, wie er in der jüngeren linguistischen Literatur meist definiert wird. Danach gehören zu einer Flexionsklasse Lexeme, deren Formen den gleichen Satz morphologischer Markierungen aufweisen (genauer: Wurzel 1984, S. 66; Carstairs-McCarthy 2000, S. 630). Nach dieser Definition könnten Neutra und Maskulina im Lateinischen nie in die gleiche Flexionsklasse fallen (Wurzel 1984, S. 54) - ihre Formenbildung unterscheidet sich ja ganz eklatant. Umgekehrt würde jeder beliebige Formenunterschied, soweit er als morphologisch relevant betrachtet wird, genügen, um Flexionsklassen zu etablieren. Den traditionellen Deklinationen entsprechen nach diesem Verständnis eher Konzepte wie das des Makroparadigmas bei Carstairs-McCarthy (Carstairs 1987, S. 87). Obwohl die Termini ,Deklinationsklasse“ und ,(nominale) Flexionsklasse' in der Literatur oft ohne deutliche Unterscheidung verwendet werden, sollte klar sein, dass die beiden Begriffe sorgfältig auseinander gehalten werden müssen. ${ }^{3}$

\subsection{Paradigmenvielfalt im Ungarischen}

Das Ungarische liefert (neben dem Türkischen) ein weiteres Beispiel für agglutinierende Sprachen, die als Sprachen ohne Deklinationsklassen gelten. Die Formenbildung erfolgt mittels Suffigierung, wobei längere Flexivketten entstehen können, wie die Beispiele in Tabelle 2 zeigen (nach Törkenczy 2002, S. 19).

\begin{tabular}{|c|c|c|}
\hline barát & Freund & ,Freund ${ }^{\varsigma}$ \\
\hline barát-ok. & Freund-PL & ,Freunde 6 \\
\hline barát-om & Freund-1SG & ,mein Freund \\
\hline barát-ai-m & Freund-PL-1SG & ,meine Freunde \\
\hline barát-ai-m-é & Freund-PL-1SG-POSS & ,der/die/das meiner Freunde \\
\hline barát-ai-m-é-i & Freund-PL-1SG-POSS-PL & ,die meiner Freunde، \\
\hline barát-ai-m-é-i-nak & Freund-PL-1SG-POSS-PL-DAT & ,denen meiner Freunde 6 \\
\hline
\end{tabular}

Tab. 2: Ungarisch: Flexivketten

Bei dieser stark synthetischen Nominalflexion nehmen Wortformen vieles auf, was in einer stärker analytischen Sprache wie dem Deutschen erst auf Nominalgruppenebene etwa mittels Possessivpronomina oder Präpositionen realisiert wird. Die Vielzahl der Flexive bringt es mit sich, dass jedes Substantiv über 700 Flexionsformen besitzt (Gergely/Pléh 1994, S. 178). Ansätze, die eine holistische Betrachtung von Wortformen favorisieren, stoßen hier auf

Thieroff (2000, S. 309) unterscheidet ,Deklinationstypen', die sich ,schematisch' aus den möglichen Kombinationen von Flexiven ergeben, und ,Deklinationsklassen', die ein oder mehrere Deklinationstypen umfassen können. Zur kritischen Diskussion der Definitionen von ,Flexionsklasse` vgl. auch Kürschner (2008, S. 20-23, 366-368 mit weiteren Literaturangaben). 
Schwierigkeiten. Selbst wenn man ein sehr häufig vorkommendes Substantiv wie EMBER (,Mann') nimmt, kann man ausschließen, dass ein Sprecher im Laufe seines Lebens alle Formen auch nur einmal hören wird. Die Sprecher können sich die fraglichen Wortformen daher auch nicht, als Ganze' aneignen (Gergely/Pléh 1994 im Anschluss an Kornai 1992). Die Flexionsformen des Ungarischen wird man sich eher als ,Output ${ }^{6}$ einer, Wortsyntax ${ }^{6}$ vorstellen. Aus dieser Sicht erscheint es nahezu trivial, dass Deklinationsklassen in einem solchen System keine Rolle spielen. Andererseits gibt es für deutschsprachige Lerner einen Band mit „74 Mustertabellen mit sämtlichen Deklinationstypen“ (Hegedűs 2006). Das auf Vollständigkeit angelegte „Wörterbuch ungarischer Flexionen“ (Elekfi 1994) kommt auf weit über 100 SubstantivParadigmen. Wie kommt es zu diesem scheinbaren Widerspruch?

Ich betrachte exemplarisch das allgemeine Pluralflexiv der Substantive. Es besteht aus einem kurzen Vokal mit mittlerem Öffnungsgrad gefolgt vom Konsonanten $k$ wie in den Beispielen (1), (2), und (3) in Tabelle 3. Welcher Vokal auftritt, richtet sich nach den Regeln der Vokalharmonie, die im Ungarischen allgemein gelten. Endet der Stamm wie in (4) auf Vokal, so fällt der Initialvokal des Flexivs aus - ebenfalls ein regulärer Vorgang. Es handelt sich hier um ein einheitliches Pluralflexiv, dessen Form aber teilweise unterspezifiziert ist. Die Notation $-(V) k /$ PL zeigt dies an.

\begin{tabular}{|c|c|c|}
\hline Singular & Plural & \\
\hline barát ,Freund“ & barát-ok. & Vokalharmonie: hinten \\
\hline sqék, Stuhl' & szék-ek & Vokalharmonie: vorn \\
\hline ör ,Wächter ${ }^{6}$ & ör-ök & Vokalharmonie: vorn, gerundet \\
\hline kocsi,Auto“ & kocsi-k & Ausfall des Flexivvokals \\
\hline cél ,Ziel' & cél-ok & antiharmonisch \\
\hline férfi,Mann“ & férfi-ak & fehlender Vokalausfall \\
\hline városi ,Städter ${ }^{6}$ & városi-ak & fehlender Vokalausfall \\
\hline csinos ,hübsch“ & csinos-ak & offener Flexivvokal \\
\hline ház, ,Haus' & báz-ak & offener Flexivvokal \\
\hline táska ,Tasche' & táská-k & $a$-Dehnung \\
\hline bokor,Busch“ & boker-ok & Synkope \\
\hline sátor ,Zelt ${ }^{6}$ & sátr-ak & Synkope, off. Fl.-vokal \\
\hline pohár,Glas‘ & pohar-ak & Kürzung, off. Fl.-vokal \\
\hline dij ,Preis' & dij-ak. & antiharmon., off. Fl.-vokal \\
\hline hid,Brücke“ & bid-ak & Kürzung, antiharm., off. Fl.-vokal \\
\hline tó ,See & tav-ak & $v$-Stamm, off. Fl.-vokal \\
\hline teher, Last $^{6}$ & terh-ek & Metathese \\
\hline
\end{tabular}

Tab. 3: Ungarisch: Pluralflexiv $-(V) k / \mathrm{PL}$ 
Das Auftreten der Flexivvarianten ist auf transparente Weise phonologisch determiniert, aber es gibt lexikalische Ausnahmen. Etwa 60 Substantive mit vorderem Stammvokal wie in (5) nehmen entgegen der Regel Flexivvarianten auf hinteren Vokal (Törkenczy 2002, S. 14, ,anti-harmonic stems“). In (6) fällt der Flexivvokal gegen die Regel nicht aus. Häufiger kommt dies bei Adjektiven und bei substantivierten Adjektiven wie in (7) vor. Die Wahl des Flexivvokals ist zudem wortartabhängig. Adjektive wie in (8) verlangen die Flexivvariante -ak (mit offenem Vokal), wo Substantive die -ok-Variante nehmen würden. Aber in beiden Richtungen gibt es Ausnahmen. (9) zeigt die -ak-Variante am Substantiv. Es gibt also eine lexikalisch zu markierende Klasse von Substantiven, die die ,falsche ${ }^{\varsigma}$ Flexivvariante nehmen.

Auch Stämme können im Ungarischen Varianten zeigen, insbesondere solche, die aus ugrischer oder uralischer Zeit ererbt sind (Abondolo 2009, S. 492). Geht der bloße Stamm auf offenen Vokal aus wie in (10), so wird der Vokal regelmäßig gedehnt, wenn an den Stamm ein Flexiv tritt. (Der Akzent zeigt Vokallänge an.) Andere Alternationen sind unproduktiv. Betroffen sind etwa 500 Stämme (ebd.), die unter anderem Synkope oder Kürzung von Stammvokalen vor Flexiven zeigen. Weitere Besonderheiten in verschiedenen Kombinationen kommen vor.

In einem schematisch konzipierten Flexionsklassenmodell werden alle diese Variationen zusammen geworfen - von phonologisch regelmäßigen Wechseln über subreguläre Prozesse bis zu lexikalischen Idiosynkrasien. Die Variablen multiplizieren sich und es kommt zur ungehemmten Proliferation der Paradigmen.

Für die ganz große Mehrheit der ungarischen Substantive steht die Pluralform aber fest, ohne dass es überhaupt lexikalischer Subklassenbildungen bedarf. Nur wo besondere Abweichungen von generellen Mustern auftreten, müssen geschlossene, lexikalisch spezifizierte Klassen identifiziert werden. Die einzelnen Quellen der Variation müssen und können je für sich und in ihrem Zusammenspiel analysiert werden. Das Ungarische weist eine enorme Vielfalt von Paradigmen (oder Flexionsklassen) auf, die ich hier nur andeuten konnte. Andererseits werden Deklinationsklassen im traditionellen Sinn nicht benötigt. Man sieht hier: Paradigmenvielfalt impliziert nicht, dass Deklinationsklassen eine tragende Rolle in der Architektur eines Flexionssystems spielen.

\subsection{Pluralbildung im Italienischen}

Im Italienischen ist die Substantivflexion auf die Pluralbildung beschränkt. Die Suffixe der auf unbetonten Vokal ausgehenden Singularformen verweisen noch auf die alten Deklinationen wie bei CAPRA, F (,Ziege), LUPO, M (,Wolf'), CANE, M (,Hund'), vgl. Tabelle 1 zum Lateinischen, und auch für das 
heutige Italienische wird diskutiert, wie eine korrekte DeklinationsklassenEinteilung vorzunehmen ist und wie viele Deklinationsklassen anzunehmen sind (D'Achille/Thornton 2003).

Tatsächlich umfasst die italienische Pluralbildung aber nur noch zwei reguläre Muster (Dardano/Trifone 1995, S. 180): Das Singularflexiv wird durch eines der beiden Pluralflexive, $-i$ oder $-e$, ersetzt. Substantive, die kein Singularflexiv besitzen, können daher keine besondere Pluralform bilden; die numerusunmarkierten Grundformen fungieren dann auch als Pluralformen. Dies gilt für Substantive, die auf Konsonant oder auf betonten Vokal ausgehen wie BAR, M (,Bar/Café) oder CITTÀ, F (,Stadt'), einschließlich Einsilbern wie RE, M (,König). Grundformen von Kurzwörtern und Fremdwörtern können als unzerlegbar behandelt werden (Repetti 2006), auch wenn sie auf Vokal auslauten, zumal wenn der auslautende Vokal bei Feminina nicht das genustypische $a$ ist, z.B. bei FOTO, F (,Foto), oder bei Maskulina nicht das genustypische $o$ wie z.B. bei CINEMA, M (,Kino $).{ }^{4}$ Die Kategorie Neutrum ist beseitigt, aber es gibt einige lexikalisierte Relikte der alten Pluralbildung des Neutrums auf $-a$ (lexikalische Plurale‘ im Sinne von Acquaviva 2008, S. 123-161). Hinzu kommen Fälle von Stammvariation im Plural durch Palatalisierung vor $-i$ und eine Handvoll Unregelmäßigkeiten.

Für die Substantive, die überhaupt besondere flexivische Pluralformen bilden, gilt nahezu ausnahmslos: Feminina, die im Singular auf $-a$ ausgehen wie SORELLA, F (,Schwester) oder PIANISTA, F (,Pianistin), bilden den Plural auf $-e$. Alle anderen bilden den Plural auf $-i$. Dazu gehören Maskulina mit Grundformen auf $-0,-a$ oder $-e$ wie TRENO, M (,Zug'), PIANISTA, M (,Pianist'), PROBlema, M (,Problem') und PADRE, M (,Vater'), ebenso wie Feminina auf $-e$ wie MADRE, F (,Mutter') und sogar das Femininum MANO, F (,Hand ${ }^{`}$ ). Die beiden Pluralflexive können wie folgt identifiziert werden:

$$
-i / \mathrm{PL} \quad-e / \mathrm{PL}_{\mathrm{F} a}
$$

Für den $e$-Plural gilt, wie die Beispiele zeigen, eine doppelte Anwendungsbedingung, die das Genus und die Singularform betrifft. In der Notation für Flexive, die ich hier verwende, erscheint die Anwendungsbedingung als Subskript (mit Fa für ,Femininum auf $-a^{\text {) }}$. Wenn die Anwendungsbedingung erfüllt ist, muss der $e$-Plural gewählt werden. Der $i$-Plural gilt sonst; er bleibt ohne Anwendungsbedingung. Das Flexiv $-i$ ist das allgemeine, unspezifische oder unmarkierte Pluralsuffix des Italienischen, das Flexiv - $e$ ein konkurrierendes, markiertes Gegenstück. Zu einem Flexiv gehört danach im Allgemeinen dreierlei: Form, Funktion und Anwendungsbedingung. Im

$4 \quad$ Der Unterschied zwischen einer ungegliederten Grundform wie foto gegenüber einer gegliederten Grundform wie tren-o lässt sich erfassen, wenn man mit Wurzel (1984, S. 56) gegliederte Lexikonrepräsentationen zulässt. 
unmarkierten Fall ist die Anwendungsbedingung leer. In einer idealen agglutinierenden Sprache kommen natürlich alle Flexive ohne Anwendungsbedingungen aus.

Die Notwendigkeit, zwischen Deklinationsklassen im traditionellen Sinn und Flexionsklassen zu unterscheiden, wird hier sehr deutlich. Rechnet man zu einer Flexionsklasse gerade solche Lexeme, deren Formen den gleichen Satz morphologischer Markierungen aufweisen, so fallen Maskulina wie TRENO, PROBLEMA und PADRE in verschiedene Klassen. Soll die Klassifikation dagegen nur die Herleitung der Flexionsformen sicherstellen, werden diese Unterscheidungen nicht benötigt. Die Pluralbildung ist ja für alle diese Substantive gleich. Bei gegebener Grundform (hier der Singularform) und unter der Voraussetzung, dass Pluralsuffixe Singularsuffixe ersetzen, ist mit der Identifizierung der beiden Flexive die reguläre Substantivflexion des Italienischen bereits vollständig erfasst. Über die mit den Grundformen ohnehin gegebenen Eigenschaften hinaus wird nichts benötigt, also insbesondere keine (im Sinne von Corbett) unabhängige Deklinationsklasseneinteilung. Man sieht hier: Eine Deklinationsklasseneinteilung, wie sie für das Lateinische benötigt wird, erweist sich für das Italienische als überflüssig, wenn man die Markiertheitsverhältnisse unter den Suffixen berücksichtigt und bei Suffixen Anwendungsbedingungen zulässt.

\subsection{Kasusflexion im Polnischen}

Im Slawischen sind die Flexionssysteme insgesamt gut erhalten, aber sie sind doch in Übereinstimmung mit der allgemeinen Entwicklungstendenz stark umgestaltet. ${ }^{5}$ Wie ich zeigen möchte, hat die Herausbildung paradigmenübergreifender Flexivinventare dazu geführt, dass die Form-FunktionsBeziehung dem Eins-zu-eins-Ideal wenigstens viel näher kommt, als dies auf älteren Sprachstufen der Fall war. Wie im Falle des Italienischen wird dies sichtbar, wenn die Markiertheitsverhältnisse und Anwendungsbedingungen berücksichtigt werden.

Das polnische Substantivsystem zeigt, folgt man Beschreibungen wie Orzechowska (1999) oder Swan (2002), eine sehr hohe Zahl von Deklinationsklassen. Ich betrachte hier einen repräsentativen Ausschnitt, nämlich die wichtigsten Paradigmen des Singulars der Maskulina und Neutra. Tabelle 4 zeigt die Flexive (ohne Darstellung von Stammwechseln). Im Maskulinum bilden die Belebten wie STUDENT (,Student') und BIOLOG (,Biologe') gegenüber CUKIER (,Zucker') und BANK (,Bank') eine besondere Klasse (M-BEL). Ferner wird zwischen Substantiven mit, weichem Stamm` wie POLE

Siehe Bräuer (1969) zu den vielfältigen „Deklinationsvereinheitlichung[en]“ (S. 19). Zum Folgenden vgl. Halle/Marantz (2006) und Wiese (2011), wo eine umfassendere Analyse der polnischen Substantivflexion gegeben wird. 
(,Feld') und solchen mit hartem Stamm unterschieden wie BIURO (,Büro) und die übrigen Beispiele. Substantive mit Stämmen, die auf Velar ausgehen wie BIOLOG, BANK und TANGO (,Tango), zeigen zusätzliche Besonderheiten. Bei den Kasus kann man zwei Gruppen unterscheiden: direkte Kasus (Nominativ, Vokativ und Akkusativ) und oblique Kasus im Sinne von Trubetzkoy (1934), nämlich Lokativ, Dativ, Genitiv und Instrumental.

\begin{tabular}{|c|c|c|c|c|c|c|c|}
\hline & $\mathrm{NOM}$ & VOK & AKK & LOK & DAT & GEN & INS \\
\hline STUDENT, M & - & '-e & $-a$ & '-e & -owi & $-a$ & $-e m$ \\
\hline CUKIER, M & - & '-e & - & '-e & -owi & $-u$ & $-e m$ \\
\hline BIOLOG, M & - & $-u$ & $-a$ & $-u$ & $-o w i$ & $-a$ & $-e m$ \\
\hline BANK, M & - & $-u$ & - & $-u$ & -owi & $-u$ & $-e m$ \\
\hline BIURO, N & -0 & -0 & -0 & '-e & $-u$ & $-a$ & $-e m$ \\
\hline TANGO, N & -0 & -0 & -0 & $-u$ & $-u$ & $-a$ & $-e m$ \\
\hline POLE, N & $-e$ & $-e$ & $-e$ & $-u$ & $-u$ & $-a$ & $-e m$ \\
\hline
\end{tabular}

Tab. 4: Polnisch: Singular der Maskulina und Neutra

Die Paradigmen zeigen massive formale Übereinstimmungen, die besonders deutlich hervortreten, wenn die Kasus wie in der Tabelle von links nach rechts, statt wie gewöhnlich von oben nach unten angeordnet werden. Anders als im Lateinischen gibt es hier keine formal ausgezeichneten ,Sätze von Flexiven', die sich als Ganze gegenüberstehen und jeweils für eine bestimmte Deklination gelten. Den polnischen Paradigmen fehlt die deutliche Ausprägung der Distinktivität von Flexivsätzen im Sinne von Corbett (2009). Fast alle Flexive treten in mehreren Paradigmen auf. Besonders auffällig ist die scheinbar gänzlich arbiträre Verteilung des Flexivs $-u$, das in jedem Paradigma in anderen Kasus (und einer anderen Zahl von Kasus) auftritt.

Man betrachte nun zunächst die Verteilung der Flexive in den obliquen Kasus (LOK, DAT, GEN, INS). Im Instrumental haben alle Maskulina und Neutra das gleiche Suffix. Hier ist sofort offensichtlich, dass die früheren Deklinationsklassenunterschiede beseitigt worden sind. Insgesamt gibt es in den obliquen Kasus fünf Flexive, die wie folgt identifiziert werden können:

$$
-u / \mathrm{OBL} \quad-e / \mathrm{LOK} \quad-\text { owi/DAT } \mathrm{DM}_{\mathrm{M}} \quad-a / \mathrm{GEN}_{* \mathrm{M}-\mathrm{UNBEL}} \quad-e m / \mathrm{INS}
$$

Innerhalb der obliquen Kasus erscheint '-e nur im Lokativ, -owi nur im Dativ, $-a$ nur im Genitiv und -em im Instrumental. Diese Flexive sind jeweils auf einen einzelnen Kasus spezialisiert, aber sie treten in den betreffenden Kasus nicht immer auf. Das Suffix -owi tritt nur im Maskulinum, nicht im Neutrum auf. Hier gibt es also - ähnlich wie beim italienischen $e$-Plural - eine 
genusbezogene Anwendungsbedingung, die die Verwendung des Flexivs einschränkt und die hier wieder durch ein Subskript angegeben wird (daher: -owi/ $\mathrm{DAT}_{\mathrm{M}}$ ). Beim Genitivsuffix - a greift ebenfalls eine genusbezogene Anwendungsbeschränkung. Im Grundsatz gilt - auch wenn Ausnahmen nicht selten sind -, dass dieses Flexiv nicht bei unbelebten Maskulina verwendet wird (im Einzelnen siehe Swan 2002, S. 71-74); daher: -a/GEN *M-UNBEL $_{\text {- }}$ Das Sternchen zeigt an, dass es sich um eine negative Anwendungsbedingung handelt.

Auch die Verwendung des Lokativflexivs ist beschränkt, aber in formaler, nicht in kategorialer Hinsicht. Das Flexiv verlangt einen Stammwechsel (von harter zu weicher Stammvariante). Der vorangestellte Apostroph ist das Zeichen dafür. Aber nicht alle Stammtypen lassen einen solchen Wechsel zu. Nur harte Stämme können erweicht werden. Auch Velarstämme machen die Erweichung in diesem Fall nicht mit. Wo Erweichung nicht zugelassen ist wie bei den Beispiellexemen POLE (weich) und BIOLOG, BANK und TANGO (velar), ist das Flexiv nicht anwendbar.

Das fünfte Suffix $(-u)$ ist dagegen nicht auf einen bestimmten Kasus spezialisiert. Es tritt in verschiedenen obliquen Kasus auf. Die Funktionsbestimmung OBL trägt dem Rechnung: $-u$ ist ein nicht weiter spezifiziertes, allgemeines Obliquesuffix, im Unterschied zu den übrigen je auf einen Kasus spezialisierten Obliquesuffixen. Damit klärt sich die scheinbar arbiträre Verteilung von $-u$. Die Verwendung der speziellen Obliquesuffixe $-e$, -owi und $-a$ ist durch die gegebenen Anwendungsbedingungen beschränkt. Überall $\mathrm{da}$, wo sie nicht verwendet werden, kommt das allgemeine Obliquesuffix $-u$ zum Zuge. Das $u$-Suffix füllt alle verbliebenen Lücken, und diese ,Lückenbüßer-Funktion' erklärt die prima facie unerklärlich erscheinende Verteilung.

Mit der oben gegebenen Identifizierung der Flexive ist auch ihre Verteilung in den obliquen Kasus bereits vollständig festgelegt. Die Behandlung der direkten Kasus (NOM, VOK, AKK) ist vergleichsweise unproblematisch. Nach einer ,Verweisregel', die sich so oder ähnlich ganz allgemein im Slawischen findet, gilt: Bei belebten Maskulina wird die Genitivform auch im Akkusativ verwendet. Im Polnischen fallen zudem bei den Maskulina Vokativ und Lokativ regelmäßig zusammen. Es gilt eine weitere Verweisregel, nach der, von einigen Ausnahmen abgesehen, das im Lokativ auftretende Flexiv bei den Maskulina auch im Vokativ verwendet wird. In allen Positionen, für die soweit noch kein Flexiv festgelegt ist, steht die Grundform, wie sie im Nominativ Singular erscheint. Sie fungiert als unmarkierte Form, die immer dann auftritt, wenn keine andere Regelung greift. Die Grundform gilt daher bei den unbelebten Maskulina auch für den Akkusativ und bei den Neutra (wie im Lateinischen und verwandten Sprachen) für alle direkten Kasus.

Es zeigt sich, dass die betrachteten Paradigmen ihre Flexive aus einem gemeinsamen Inventar beziehen. Die Anwendungsbedingungen der verschiedenen Flexive (und der Verweisregeln) unterscheiden sich und daher 
variiert auch die Verteilung von Paradigma zu Paradigma. Sie steht jedoch fest, wenn das Flexiv- (und Regel-) Inventar gegeben ist. Jedes Flexiv besitzt eine eindeutig charakterisierte Funktion und gegebenenfalls eine Anwendungsbedingung. An die Stelle eines Systems distinkter Flexivsätze für verschiedene Deklinationsklassen wie im älteren Slawischen ist ein paradigmenübergreifendes Flexivinventar getreten. Bei gegebenem Flexivinventar erweisen sich Deklinationsklassenunterscheidungen als überflüssig. Man sieht hier: Die Aufhebung von Deklinationsklassenunterscheidungen findet nicht nur bei Verlust der Kasusflexion statt, sondern kann auch in Sprachen mit gut erhaltener Kasusflexion eintreten.

\section{Deklinationsklassen und die deutsche Substantivflexion}

\subsection{Flexionsformen}

Im Folgenden gebe ich einen kurzen Abriss der deutschen Substantivdeklination. ${ }^{6}$ Im Mittelpunkt steht die Identifizierung der Flexive, die bei Substantiven im Gegenwartsdeutschen auftreten. Substantive besitzen ein festes Genus (M, F, N) und zeigen Formenvariation bezüglich Numerus (SG, PL) und Kasus (NOM, AKK, DAT, GEN). Traditionell wird die Formenbildung der Substantive mit Hilfe von Paradigmen dargestellt, die acht Positionen für die acht möglichen Kasus-Numerus-Kombinationen umfassen. Die Anzahl der formal tatsächlich unterschiedenen Flexionsformen bei Substantiven liegt jedoch nur zwischen zwei und fünf. Die Diskrepanz zwischen der Zahl der Kategorienkombinationen und der Zahl der Formen hat systematischen Charakter und muss in einer angemessenen Analyse der Substantivflexion von vornherein berücksichtigt werden. Dies ist in traditionellen Darstellungen nicht geschehen.

Der Bau der Substantivformen kann zunächst anhand eines repräsentativen Beispiels erläutert werden. Das Substantiv HAUS, N, besitzt vier Formen: Haus, Hauses, Häuser, Häusern. ${ }^{7}$ Der unmodifizierte Stamm Haus fungiert als Grundform. Die Grundform tritt insbesondere im Nominativ Singular auf, aber sie ist nicht auf einen bestimmten Kasus festgelegt. Sie fungiert vielmehr als allgemeine, d.h., kasusunspezifische Singularform und

Eine Diskussion der vielfältigen Analysen in der außerordentlich umfangreichen Literatur ist hier nicht möglich (siehe dazu u.a. Bittner 1991 und Thieroff 2000). Die folgende Darstellung zielt nicht darauf ab, neue Vorschläge zu strittigen Einzelfragen zu präsentieren, sondern ordnet mehr oder weniger gut bekannte Befunde (vgl. die zusammenfassende Darstellung in der Duden-Grammatik 2009) in ein aus dem Sprachvergleich zu rechtfertigendes Gesamtbild neu ein. Fremdwörter und Eigennamen bleiben außer Betracht.

Ferner existiert die Form Hause, DAT.SG. Formen dieses Typs gelten heute als weitgehend obsolet. Ihre Verwendung ist an besondere stilistische Kontexte oder feste Wendungen gebunden. Für die Zwecke der vorliegenden Untersuchung können sie außer Betracht bleiben. 
tritt auch im Akkusativ und im Dativ auf. Kasusspezifische Singularformen werden auf der Basis der Grundform mit Hilfe von Kasusflexiven gebildet. Die Anwendung des Genitivflexivs liefert die Genitiv-Singular-Form Hauses. Die kasusunspezifische Singularform Haus deckt alle Singularkasus ab, für die keine kasusspezifischen Formen zur Verfügung stehen (Nominativ, Akkusativ, Dativ).

Von der Grundform Haus wird mittels eines Pluralflexivs die Form Häuser gebildet. Diese Form fungiert als allgemeine Pluralform, die insbesondere im Nominativ Plural auftritt, aber wie die Grundform nicht auf einen bestimmten Kasus festgelegt ist. Sie deckt alle Kasus des Plurals ab, soweit nicht kasusspezifische Formen zur Verfügung stehen. Dies ist nur im Dativ der Fall. Von der allgemeinen Pluralform Häuser wird durch Anwendung eines Kasusflexivs die Dativ-Plural-Form Häusern abgeleitet. ${ }^{8}$

Allgemein gilt: Gewöhnliche Substantive des Deutschen (wie HAUS) besitzen zwei kasusunspezifische Formen. Ich bezeichne sie im Folgenden als Stammformen (hier: Haus, Häuser). ${ }^{9}$ Die Stammformen treten als Wortformen auf, fungieren aber zugleich auch als Stämme, auf deren Grundlage kasusspezifische Formen abgeleitet werden, soweit solche gebildet werden. Kasusspezifische Formen, die von den Stammformen mittels Kasusflexiven abgeleitet werden, bezeichne ich im Folgenden kurz als Kasusformen (hier: Hauses, Häusern). Die folgende Analyse der deutschen Substantivflexion muss danach zum einen die Bildung der Stammformen und zum anderen die Bildung der Kasusformen erfassen. Vorauszuschicken ist eine Bemerkung zur Form der Flexive.

\subsection{Flexivvarianten}

Substantivflexive bestehen im Deutschen aus einem Vokal (z.B. Tag-e) oder aus einer Folge von Vokal und Konsonant (z.B. Mann-es) oder, in einem Fall, aus einem einfachen Konsonanten (z.B. Auto-s). Der auftretende Vokal ist immer Schwa (///). Ähnlich wie im Ungarischen ist der Initialvokal der Flexive instabil. Entsprechend ergeben sich schwahaltige und schwalose (oder silbische und unsilbische) Varianten. Die übliche Notation (z.B., - (e) oder $-(e) n$ mit einem in Klammern gesetzten Vokalzeichen) zeigt das an. Die Verteilung der Varianten hängt von verschiedenen Faktoren ab und lässt auch Schwankungen und freie Variation zu. Eine detaillierte Erörterung ist

8 Die vier Formen von HAUS bilden zusammen genommen ein unterspezifiziertes Paradigma im Sinne von Wiese (1992). Die Ableitung vollständiger Paradigmen erfordert neben dem Spezifizitätsprinzip keine weiteren Annahmen; vgl. Wiese (2004), am Beispiel des Russischen. Eine Analyse der deutschen Substantivflexion unter Verwendung unterspezifizierter Paradigmen gibt Eisenberg (1998, S. 162); vgl. auch Gallmann (1998).

9 Ich folge damit Fourquet (1952, S. 33), der für die gewöhnlichen Substantive wie z.B. SOHN „un radical de singulier“ (Sobn-) und „un radical de pluriel“" (Söbne-) ansetzt. 
im vorliegenden Zusammenhang entbehrlich, doch muss der folgende Gesichtspunkt erwähnt werden. Definitiv ausgeschlossen sind schwahaltige Flexivvarianten bei Substantiven, wenn der Stamm bereits auf Schwa oder Schwa+Sonorant ausgeht wie in Katze oder Gebirge und in Messer oder Segel (,Schwa-Regel für Substantive) (Curme 1922, S. 71 f.; Bech 1963, S. 178). ${ }^{10}$ Beispielsweise lautet der mit dem Flexiv -(e)s gebildete Genitiv Singular von SEGEL aufgrund der Wirkung dieser Regel Segels, niemals * Segeles. Ebenso lautet die Pluralform zu SCHWESTER, die mit dem Pluralflexiv -(e)n gebildet wird, Schwestern, nicht: *Schwesteren. Bei bloßen Schwa-Flexiven, also Flexiven ohne konsonantischen Bestandteil, bewirkt die Regel, dass die Suffigierungsoperation gegebenenfalls ganz oder teilweise leerläuft. Die zu erwartende Pluralbildung mit dem Pluralflexiv -(e) liefert für SEGEL die Pluralform Segel, die ohne äußeres Pluralkennzeichen bleibt (,Nullplural'). Das Gleiche gilt für GEBIRGE (*Gebirge-e). Bei der Pluralform Mütter zu MUTTER (nicht: *Müttere) beschränkt sich aus dem gleichen Grund der Effekt der Anwendung des Pluralflexivs auf den Umlaut (vgl. dagegen Hände, Plural zu HAND). Umgekehrt müssen Pluralsuffixe in der schwahaltigen Variante erscheinen, wenn dies nicht durch die Schwa-Regel ausgeschlossen ist (daher Hände, aber nicht: *Händ).

Weitere Fälle (ganz oder teilweise) leerlaufender Anwendung von Flexiven ergeben sich bei der Anwendung umlautender Pluralbildung auf Stammformen mit nicht umlautfähigem Vokal wie bei Kinder zu KIND (gegenüber Häuser zu HAUS) sowie dann, wenn die Suffigierung der schwalosen Variante eines Flexivs eine (im Deutschen ausgeschlossene) Geminate liefern würde. So käme bei der Anwendung von -(e)n auf Stammformen wie Wagen oder Schwestern wegen der Schwa-Regel für Substantive nur die schwalose Variante in Betracht, deren Verwendung eine phonotaktisch ausgeschlossene Form liefern würde $(* W$ agen-n, $*$ S chwestern- $n)$ (,Geminatenverbot $\left.{ }^{\top}\right)$. Hier unterbleibt die Suffigierung ganz (Curme 1922, S. 72; Bech 1963, S. 183). Umgekehrt ist regelmäßig die schwahaltige Variante des Flexivs gefordert, wo sie zulässig ist, wenn die Setzung der schwalosen Variante eine ausgeschlossene Form liefern würde. Zu HAUS lautet der Genitiv Singular daher Haus-es, nicht: *Haus-s); Unterlassung der Suffigierung wird hier (außer bei Fremdwörtern) gewöhnlich nicht geduldet (Duden-Grammatik 2009, S. 203 f., Rdnr. 312).

$10 \quad$ Zur genaueren Diskussion der beschriebenen Regularität siehe u.a. Steche (1927, S. 96 et passim) und Neef (1998, mit Literaturhinweisen). Sie wird manchmal als besondere Pluralregel präsentiert (Duden-Grammatik 2009, S. 181, Grundregel 3) und mit einer vermuteten Bevorzugung trochäischer Pluralformen in Verbindung gebracht (Eisenberg 1998, S. 159). Es muss daher hier betont werden, dass die Regel nicht pluralspezifisch ist - sie gilt auch im Singular. 


\subsection{Pluralstammformen}

Für die Bildung der Pluralstammformen der deutschen Substantive stehen fünf Flexive zur Verfügung. Im Bereich der gewöhnlichen Substantive dominieren zwei Pluralflexive, die wie folgt identifiziert werden können:
$-(e) / \mathrm{PL}$
$-(e) n / \mathrm{PL}_{\mathrm{F}}$

Beispielformen sind Hunde (zu HUND, M), Schafe (zu SCHAF, N) oder Katzen (zu KATZE, F). Wie zuvor sind die Flexive durch die Verknüpfung von Form, Funktion und Anwendungsbedingung gekennzeichnet. (Die Anwendungsbedingung kann wie immer leer sein.) Die Verteilung der Pluralflexive ist durch das Genus bestimmt (Augst 1979, S. 224). ${ }^{11}$ Das markantere, konsonantische Flexiv auf Nasal erscheint bei Feminina, bei denen schon die Singularstammform häufig auf Schwa auslautet (wie bei Katze). Sonst (bei Maskulina und Neutra) steht das bloße Schwa-Flexiv, formal das minimale Flexionskennzeichen des Deutschen, das damit den schlechthin unmarkierten Fall der Pluralbildung im Deutschen darstellt. Die Verteilung wird durch die Anwendungsbedingung für das Nasalflexiv erfasst. Die Formenbildung mittels dieser beiden Flexive konstituiert das Normalmuster der Pluralbildung der Substantive im Gegenwartsdeutschen (,Normalplural ${ }^{\top} .^{12}$

Wie am Beispiel des Ungarischen erwähnt, treten Abweichungen von den regulären Mustern der Formenbildung insbesondere bei altüberlieferten Substantiven auf und sind daher im Grundwortschatz am ehesten zu erwarten. Hier muss mit unregelmäßigen, auf geschlossene Klassen beschränkten Stammalternationen gerechnet werden. Im Deutschen existieren neben den bloß suffigierenden Flexiven des Normalplurals in der Gegenwartssprache unproduktive Pluralflexive (Eisenberg 1998, S. 158), deren Anwendung über die Suffigierung hinaus mit einem Wechsel des Stammvokals (Umlaut) verbunden ist wie in den angeführten Beispielen Häuser und Hände. Die mit Umlaut verbundene Pluralbildung kann als verstärkte Pluralbildung charakterisiert werden (Wiese 2000, S. 149).

$11 \quad$ Zur Unterscheidung Non-Feminina/Feminina als oberstes Einteilungsprinzip für die nhd. Deklination siehe Bittner (1991, 1994), aber auch schon Paul (1917, S. 5). Ich betrachte das Genus der Substantive hier als gegeben. Umgekehrt kann aus dem Vorliegen eines genusgebundenen Flexivs (und indirekt ebenso aus dem Vorliegen eines nicht genusgebundenen Flexivs) ein Rückschluss auf das Genus des Lexems gezogen werden. Die Möglichkeit solcher Rückschlüsse ist kein hinreichender Grund, die Deklinationsklassenzugehörigkeit als primär gegenüber dem Genus anzusehen, wie es Corbett (1982, S. 227) ausgehend vom Russischen tut.

12 Hierunter fallen auch Substantive wie MESSER oder SEGEL (mit den Pluralformen Messer und Segel), bei denen die Pluralbildung nach der erörterten Schwa-Regel für Substantive ohne sichtbaren Effekt bleiben muss. 
Das gewöhnliche Suffix bei Umlautplural ist wieder non-konsonantisch - ein bloßes Schwa-Suffix wie bei Bäume oder Hände. Daneben existiert ein konsonantisches Pluralflexiv, das, falls möglich, immer mit Umlaut verbunden ist, nämlich -er. Auch bei den mit Umlaut verbundenen Pluralbildungen liegt eine genusgebundene Verteilung vor. Das einfache Schwa-Flexiv tritt bei umlautenden Neutra nur ganz ausnahmsweise auf - sie verlangen das Flexiv -er, das daher als neutrumspezifisch angesehen werden kann. Die Zuweisung einer entsprechenden Anwendungsbedingung trägt dem Rechnung. Die regelmäßig mit Umlaut verbundenen Pluralflexive (gekennzeichnet durch vorangestelltes UL) können danach wie folgt identifiziert werden: ${ }^{13}$

$$
\mathrm{UL}_{-}(e) / \mathrm{PL} \quad \mathrm{UL}_{-}-e r / \mathrm{PL}_{\mathrm{N}}
$$

Zu den genusbezogenen Verteilungen der Pluralflexive gibt es eine Anzahl von Ausnahmen. Einige Maskulina und Neutra wie STRAHL und BETT nehmen das feminintypische Nasalflexiv. Solche Lexeme müssen lexikalisch besonders ausgezeichnet werden. Ich verwende hier die Notation , $\mathrm{M} \mid \mathrm{F}^{6}$ für ,Maskulinum, das den Plural wie ein Femininum bildet' oder kürzer: ,Maskulinum mit femininer Pluralbildung' und entsprechend in vergleichbaren Fällen. So ergibt sich zu BETT, N|F, die Pluralform Betten. Zu solchen Abweichungen gegenüber der gewöhnlichen Korrelation von Genus und Pluralbildung kann es unter anderem dann kommen, wenn ein Substantiv das Genus wechselt, aber dennoch die markantere konsonantische Pluralbildung bewahrt wie im Falle von STRAHL, M F, mittelhochdeutsch ein Femininum. Dass umgekehrt gegen die Regel das weniger markante Suffix auftritt, kommt nur ganz vereinzelt vor. Bei KENNTNIS, F $\mid \mathrm{N}$, und MÜHSAL, F $\mid \mathrm{N}$, geben die Affixe -nis und -sal, die gewöhnlich Neutra bilden, den Ausschlag (Kenntnisse, Mühsale); vgl. Fleischer (1974, S. 158 f.).

Auch im Bereich der Substantive mit umlautender Pluralbildung gibt es entsprechende Abweichungen gegenüber der gewöhnlichen Korrelation von Genus und Pluralbildung (Paul 1917, S. 30-33, 192). Eine Handvoll Maskulina nimmt den er-Plural (vgl. Götter zu GOTT, M|N); die umgekehrte Abweichung tritt wiederum nur ganz vereinzelt auf (z.B. FLOSS, N|M, Plural: Flöße).

Substantive, die aus irgendeinem Grunde einen Sonderstatus haben, wie Fremdwörter, Kurzwörter, substantivierte Indeklinabilia, phonologisch oder aus anderen Gründen , auffällige` oder ,uneigentliche` Substantive wie SNOB,

Wie bemerkt, kann auch bei diesen Flexiven die Anwendung teilweise leerlaufen (nur Umlaut bei Mütter, nur Suffigierung bei Kinder); solche Bildungen sind völlig regelmäßig und begründen keine eigenen Pluraltypen. Wie Thieroff (2009) zeigt, muss der umlautende Plural auch bei den Maskulina im Vergleich zum nicht-umlautenden Plural als die markierte Option angesehen werden (entgegen Wurzel 1984, S. 74). 
LOK, AUTO, SOFA oder A (Name des Buchstabens), bilden den Plural häufig mit dem Flexiv -s (Bornschein/Butt 1987). ${ }^{14} \mathrm{Zu}$ den Besonderheiten des $s$-Plurals (oder Sonderplurals) gehört zum einen das Fehlen einer silbischen Variante, zum anderen das Faktum, dass die mit diesem Flexiv versehenen Formen abgeschlossene Wortformen sind: Ein Kasussuffix kann nicht mehr antreten. Das Kreuzzeichen in der folgenden Notation weist darauf hin:

$$
-s \# / \text { PL }
$$

Während der Umlautplural unproduktiv ist und vor allem im Altbestand des Grundwortschatzes zu finden ist, ist der $s$-Plural sehr produktiv, findet sich aber gerade bei peripheren oder neuen Wörtern. Man hat es also mit zwei entgegengesetzten Polen der Abweichung vom Normalmuster der Pluralbildung zu tun.

Die Pluralbildung der deutschen Substantive ist komplex. Was die Besonderheit des deutschen Pluralsystems ausmacht, kann durch Vergleich mit dem viel einfacheren italienischen System herausgestellt werden. In beiden Sprachen steht im Zentrum ein Paar aus einem unmarkierten Pluralflexiv ohne besondere Anwendungsbedingungen und einem markierten, für die Verwendung bei Feminina spezifizierten Pluralflexiv, hier -(e) und $-(e) n$, im Italienischen $-i$ und $-e$. Die Ähnlichkeit der Konstellation ist natürlich nicht zufällig - sie weist ja, teils sehr vermittelt, auf die gleiche indoeuropäische Deklinationsklassenunterscheidung zurück (im Lateinischen: 2. vs. 1. Deklination). In beiden Sprachen werden diese Flexive im Allgemeinen nicht auf Lexeme angewandt, denen aus verschiedenen Gründen ein Sonderstatus zukommt: Im Deutschen kommt hier der $s$-Plural in Betracht, im Italienischen können solche Lexeme unflektiert bleiben (ein Verfahren, das auch in den slawischen Sprachen gängig ist). Beiden Verfahren ist gemeinsam, dass in die Substanz der Grundform nicht eingegriffen wird - trivialerweise, wenn diese unverändert bleibt, aber ebenso bei nicht-silbischer Suffigierung, die, anders als der Normalplural, auch durch Auslautverhärtung bedingte Stammvariation oder Resyllabierung ausschließt (Wegener 1995, S. 23). Schließlich gibt es in beiden Sprachen geschlossene Klassen, die besondere Bildungen zeigen: im Deutschen umlautenden Plural, im Italienischen den sehr viel selteneren lexikalischen $a$-Plural. Was die deutsche Pluralbildung auszeichnet, ist zum einen der relativ große Umfang der Substantive, die den unproduktiven Mustern des Umlautplurals folgen; zum anderen die Existenz einer nicht ganz kleinen Anzahl von Lexemen, die nicht die Pluralbildung zeigen, die ihr Genus erwarten ließe (wie BETT, N|F, oder GOTT,

$14 \quad$ Marcus et al. (1995) werten den s-Plural als „default plural“ (S. 231), jedoch stellt Wunderlich (1999) klar, dass der $s$-Plural auf ,atypical nouns“ beschränkt ist und dass u.a. der $n$-Plural bei Feminina als ,regular“ angesehen werden muss. 
$\mathrm{M} \mid \mathrm{N})$. Im Italienischen finden sich vergleichbare Fälle, unerwarteter ${ }^{6}$ Pluralbildung nur ganz vereinzelt wie bei den Feminina ALA (,Flügel ${ }^{\circ}$ ) und ARMA (,Waffe ${ }^{\circ}$ ) mit $i$-Plural trotz Singular auf $-a$.

\subsection{Kasusformen}

Die Kasusflexion der deutschen Substantive kann nicht losgelöst von den Verhältnissen in Nominalgruppen als Ganzen analysiert werden. Sie stellt nur eine untergeordnete Komponente im Zusammenspiel flexivischer Markierungen innerhalb von Nominalgruppen dar (vgl. Wurzel 1984, S. 90-97). Tabelle 5 zeigt die Flexive, die bei gewöhnlichen dreigliedrigen Nominalgruppen am Pronomen, Adjektiv und Substantiv auftreten, ausgenommen die oben besprochenen Pluralflexive der Substantive. Beispiele für Flexionsformen erscheinen am Fuß der Tabelle.

Pronomen

$$
\text { M N F }
$$

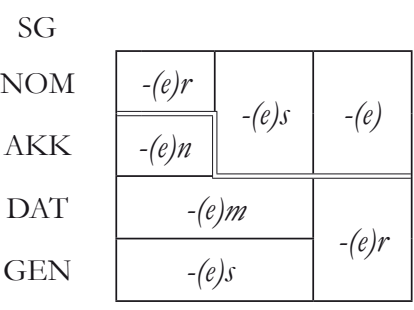

PL

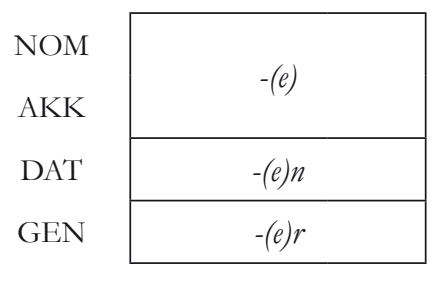

NOM.SG.M

dies-er

GEN.SG.M

NOM.PL.M

DAT.PL.M
Adjektiv
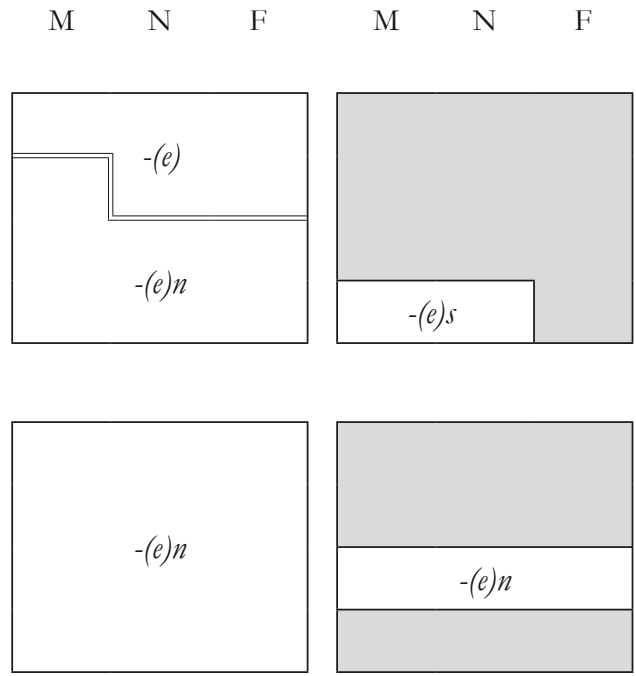

alt-e

alt-en

alt-en

alt-en
Substantiv

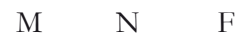

F

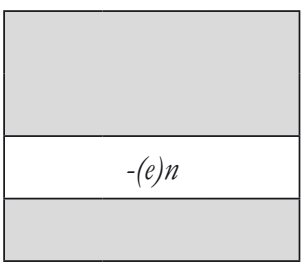

Mann

Mann-es

Männer

Männer-n

Tab. 5: Deutsch: Flexive in Nominalgruppen

Tabelle 5 veranschaulicht, dass die Hauptlast der Flexion innerhalb der Nominalgruppe auf dem Pronomen liegt, hier gezeigt am Beispiel des Demonstrativums DIESER. Bei den Grundformen (also den für Kasus und Numerus unmarkierten Formen, die im Nominativ Singular erscheinen) 
werden die drei Genera (M, N und F) unterschieden. Im Neutrum und Femininum gelten die Grundformen auch für den Akkusativ. (In der Tabelle stehen die betreffenden Flexive oberhalb der doppelten Linie.) Ein besonderes Akkusativflexiv gibt es nur im Maskulinum Singular. Besondere Kasusflexive gibt es ferner für den Dativ und den Genitiv, jedoch fallen diese Kasus im Femininum Singular zusammen. Im Plural ist die Genusunterscheidung aufgehoben. Die pronominale oder starke Flexion zeigt noch deutlich Züge des älteren indoeuropäischen Modells, etwa die formale Unterscheidung von Maskulinum und Neutrum im Nominativ und Akkusativ Singular bei gleichzeitigem Synkretismus dieser Kasus im Neutrum. Deklinationsklassenunterschiede sind aber vollständig beseitigt.

Das Adjektiv folgt in Nominalgruppen des betrachteten Typs der so genannten schwachen Flexion, die nur zwei Flexive, -(e) und -(e)n, umfasst. Das einfache Schwa-Flexiv tritt im Nominativ Singular aller Genera auf und im Akkusativ Singular, wo dieser systematisch mit dem Nominativ Singular zusammenfällt (im Neutrum und im Femininum). Das Nasalflexiv erscheint in allen übrigen Positionen, also in allen Positionen, die überhaupt für Kasus und/oder Numerus markierbar sind. Das Nasalflexiv der schwachen Deklination ist also ein allgemeines Kasus-Numerus-Kennzeichen ohne weitere Spezialisierung (vgl. Wiese 2000, S. 143).

Substantive wie MANN folgen der starken Flexion, wenn auch im Vergleich zum Pronomen einer drastisch reduzierten Variante. Aus dem Gesamtinventar der Kasusflexive der starken Flexion, das bei der Formenbildung der Pronomina verwendet wird, tritt am Substantiv im Singular nur ein einziges auf, und zwar das markanteste, gemessen am Grad der konsonantischen Stärke, nämlich das sibilantische Genitivflexiv -(e)s. ${ }^{15} \mathrm{Im}$ Plural werden Kasusformen der Substantive auf der Basis der Pluralstammformen mittels Kasusflexiv gebildet. Wie im Singular stellt die Kasusflexion der Substantive im Plural eine reduzierte Variante der starken Flexion dar. Wiederum tritt am Substantiv nur ein einziges Kasusflexiv auf, und zwar wieder dasjenige mit der größten konsonantischen Stärke, in diesem Fall das nasale Dativflexiv -(e)n. Das Gesamtinventar der am Substantiv auftretenden produktiven Kasusflexive stellt eine sehr beschränkte Teilmenge der Flexive der pronominalen oder starken Flexion dar, nämlich:

$$
-(e) s / \mathrm{GEN}_{* \mathrm{~F}, \mathrm{PL}} \quad-(e) n / \mathrm{DAT}_{\mathrm{PL}}
$$

Damit ist die Bildung der Kasusformen der Substantive (im oben bestimmten Sinn) bereits vollständig erfasst. Die Anwendungsbedingungen der betref-

15 Zum Begriff der konsonantischen Stärke (abnehmende Sonorität) siehe Vennemann (1982). Zur Bedeutung der konsonantischen Stärke für den Bau der deutschen Nominalflexion siehe Wiese (2009, S. 178-182). 
fenden Flexive sind bei den Substantiven dieselben wie bei den Pronomina (vgl. Müller 2002, S. 128): Das Genitivflexiv -(e)s ist auf den Singular der Maskulina und Neutra beschränkt, anders gesagt, seine Verwendung im markierten Genus Femininum und im markierten Numerus Plural ist ausgeschlossen (angezeigt durch: ${ }^{*} \mathrm{~F}$ und ${ }^{*} \mathrm{PL}$ ). Das Dativflexiv ist dagegen auf den Plural beschränkt (angezeigt durch: PL). Es unterliegt keinen genusbezogenen Anwendungsbedingungen.

Das Dativflexiv -(e)n/DAT ${ }_{\mathrm{PL}}$ kann bei Substantiven nach der angeführten Schwa-Regel immer nur in der schwalosen Variante erscheinen, da immer eine Schwa-Silbe vorausgeht, wie in Segel-n oder Männer-n. (Die schwahaltige Variante dieses Flexivs erscheint dagegen am Pronomen, vgl. dies-en Männer-n, DAT.PL). Wo es an eine nasal auslautende Stammform antreten würde, muss die Anwendung der schwalosen Variante des Flexivs, wie oben bemerkt, wegen des Ausschlusses von Geminaten aber leerlaufen.

Das Dativflexiv -(e)n/DAT $\mathrm{PL}_{\mathrm{PL}}$ tritt ferner nicht an Pluralstammformen, die mit dem Sonderplural (s-Plural) gebildet sind. Paul (1917, S. 129) verweist in diesem Zusammenhang auf die Kasuslosigkeit der Substantive des Französischen, das er als Quelle des s-Plurals ansieht. Angesichts der bei Fremdwörtern grundsätzlich bestehenden Neigung zur Nicht-Auszeichnung von Kasusformen, sollte es jedenfalls nicht überraschen, dass eine insbesondere für Fremdwörter verwendete Pluralbildung keine Kasussuffigierung zulässt. ${ }^{16}$

\subsection{Starke und schwache Flexion}

Traditionell stellt die Unterscheidung von starker und schwacher Deklination die Haupteinteilung in der nominalen Flexion des Deutschen dar. In den im vorangehenden Abschnitt betrachteten Beispielen für dreigliedrige Nominalgruppen werden das Pronomen und das Substantiv stark, das Adjektiv dagegen schwach flektiert. Die Unterscheidung zwischen starker und schwacher Deklination korreliert also mit den Wortarten, aber sie ist nicht fest an sie gebunden. Das Adjektiv übernimmt die starke Flexion, wenn (und insoweit) starke Suffixe andernfalls in der Nominalgruppe fehlen würden (vgl. aber im Einzelnen Wiese 2009). Bei den Substantiven folgt eine Teilklasse der Maskulina (wie MENSCH, M, oder AFFE, M) dem im Übrigen adjektivtypischen schwachen Flexionsmuster. Das bestimmende Charakteristikum der schwachen Substantivflexion liegt also in der grundsätzlichen Übereinstimmung mit der schwachen Adjektivflexion (Grimm 1837, S. 461). Nur fehlt beim Substantiv die adjektivtypische Unterscheidung zwischen

16 Nach anderer Auffassung soll das Dativ-Suffix der Substantive ein obligatorisch nicht-silbisches Suffix sein $(-n)$, so dass eine Suffigierung nach sibilantischem Wortausgang phonotaktisch ausgeschlossen wäre (*Autosn), so u.a. Eisenberg (1998, S. 161). 
unflektierter Form und unmarkierter flektierter Form (vgl. alt vs. alte). Ob die Grundform auf Schwa ausgeht, ist beim schwachen Substantiv lexikalisch geregelt (vgl. Mensch vs. Affe). Das Inventar der Flexive der schwachen Substantivflexion umfasst daher gerade ein einziges Flexiv mit der Form -(e)n, das wie beim Adjektiv als unspezifisches, nicht weiter festgelegtes Kasus-Numerus-Markiertheitszeichen fungiert.

Im Sinne der oben getroffenen Unterscheidung von Stammformen und Kasusformen besitzen schwache Substantive wie MENSCH keine Kasusformen, d.h., keine Formen, die durch Kasusflexive auf bestimmte Kasus festgelegt sind. Vielmehr gilt: schwache Flexion ist Stammformenbildung. ${ }^{17}$ Unter dieser Annahme kann man die Stammformenbildung der gewöhnlichen (starken) Substantive und der schwachen Substantive wie in Tabelle 6 vergleichen.

\begin{tabular}{cccc} 
& \multicolumn{2}{c}{ STARK } & SCHWACH \\
\cline { 2 - 4 } & MANN & FRAU & MENSCH \\
\hline $\begin{array}{c}\text { 1. Stammform, } \\
\text { unmarkiert }\end{array}$ & Mann & Fran & Mensch \\
\hline $\begin{array}{c}\text { 2. Stammform, } \\
\text { markiert für: }\end{array}$ & $\begin{array}{c}\text { Männer } \\
\text { Numerus }\end{array}$ & $\begin{array}{c}\text { Frauen } \\
\text { Numerus }\end{array}$ & $\begin{array}{c}\text { Menschen } \\
\text { Kasus-Numerus }\end{array}$ \\
\hline
\end{tabular}

Tab. 6: Deutsch: Stammformen

Gewöhnliche Substantive wie MANN oder FRAU besitzen, wie dargelegt, eine einfache und eine abgeleitete Stammform (Mann, Männer, Frau, Frauen). Die abgeleitete Stammform fungiert als markierte Numerus-Stammform (d.h., sie liegt allen Pluralformen zugrunde), im Übrigen (also im Singular) wird die einfache Stammform verwendet. Ein schwaches Substantiv wie MENSCH besitzt ebenfalls eine einfache und eine abgeleitete Stammform (Mensch, Menschen). Anders als bei den gewöhnlichen Substantiven fungiert die abgeleitete Stammform aber nicht als numerusmarkierte Stammform, sondern als Kasus-Numerus-markierte Stammform, d.h., sie deckt alle Kasus-Numerus-Kombinationen ab, ausgenommen die bezüglich Kasus und Numerus unmarkierte Kombination (also Nominativ Singular). Im Übrigen (hier also nur im Nominativ Singular) wird wieder die unmarkierte Stammform verwendet.

Diese Stammformenverteilung geht bekanntlich auf den schon indoeuropäischen Abfall des auslautenden Nasals im Nominativ Singular zurück (Paul 1989, S. 202, \186), wie er sich auch bei Substantiven der 3. Deklina-

17 Ich folge hier wieder Fourquet (1952, S. 33), der für die schwachen Substantive „un radical propre au nominatif singulier“ und „un radical terminé par $-n$ aux sept autres cas" ansetzt. Vgl. auch Antonsen (1973). 
tion im Lateinischen (z.B., homo/hominis) findet. Auch in nicht-indoeuropäischen Sprachen finden sich häufig entsprechende Stammformenwechsel zwischen Nominativ (bzw. Absolutiv) und den übrigen Kasus (Lehmann/ Moravcsik 2000, S. 738). Beispiele aus dem Ungarischen zeigt Tabelle 3, oben, z.B. TÁSKA (,Tasche') mit der ersten Stammform táska, die als Nominativ-Singular-Form fungiert, und der zweiten Stammform táská (mit Dehnung des auslautenden Vokals), die allen übrigen Flexionsformen zugrunde liegt (z.B., táskák, PL).

Bei den starken Substantiven werden auf der Basis der Stammformen besondere Kasusformen gebildet, sofern die einschlägigen Bedingungen erfüllt sind. MANN besitzt zwei Kasusformen (Mannes, Männern). FRAU besitzt keine Kasusformen (das Genitivflexiv wird bei Feminina nicht verwendet, und die Anwendung des Dativflexivs müsste leerlaufen).

Bei den schwachen Substantiven findet Kasusformenbildung nur ausnahmsweise statt. Als Basis für spezielle Kasusformen des Dativs oder Genitivs müsste die zweite Stammform dienen (z.B. Menschen), die für kasusmarkierte Formen gefordert ist (während die erste Stammform auf den Nominativ Singular beschränkt ist). Da die zweite Stammform auch hier auf Schwa + Nasal auslautet, würde die Anwendung des Dativsuffixes -(e)n wiederum leerlaufen. Die Anwendung des Genitivsuffixes -(e)s wäre formal möglich, aber sie tritt nur bei sehr wenigen Lexemen auf, etwa bei NAME, mit der Form Namens, GEN.SG. Diese im Frühneuhochdeutschen häufigere Bildung ist auf dem Weg zur Gegenwartssprache weitgehend beseitigt worden. So ist bei Substantiven wie GARTEN (aus mhd. GARTE, Paul 1989, S. 202, \187) der Stammformenwechsel, der die schwache Flexion kennzeichnet, ausgeglichen worden. Die nasalauslautende Stammform erscheint nun auch im Nominativ Singular. Damit ist der Übergang in die starke Flexion vollzogen. Sieht man von den verbliebenen Fällen im Übergangsbereich zur starken Flexion ab, so kann man in Hinblick auf die schwachen Substantive festhalten, dass an Kasus-Numerus-markierte Stammformen im Gegenwartsdeutschen keine Kasusflexive antreten. Hier setzt sich offenbar die für diese Klasse kennzeichnende Übereinstimmung mit der (schwachen) Adjektivflexion durch.

Das Flexiv der schwachen Substantivflexion kann auf verschiedene Weise charakterisiert werden. Ich nehme hier eine Anwendungsbedingung an, die die Verwendung im Nominativ Singular ausschließt (notiert als: *NOM.SG). Die Funktionsbestimmung ist leer. Das Flexiv ist daher für alle Kasus-Numerus-Kombinationen zu verwenden, ausgenommen die ausgeschlossene. Daher:

$$
-(e) n_{* \text { NOM.SG }}
$$




\subsection{Stammklassen}

Im Vorhergehenden habe ich das Inventar der Flexive beschrieben, die bei Substantiven im Gegenwartsdeutschen auftreten. Einen Überblick bietet Tabelle 7. Die Tabelle zeigt zugleich die lexikalischen Einteilungen (Stammklassen), die neben dem Genus benötigt werden, um die Verteilung der Flexive zu erfassen.

Substantivflexive dienen der Bildung von Stammformen oder von Kasusformen. Sofern die Suffigierung nicht leerläuft, besitzt jedes Substantiv zwei Stammformen, eine unmarkierte erste Stammform (z.B. Hund, Haus, Auto, Mensch) und eine markierte zweite Stammform. Die markierte Stammform fungiert entweder als numerusmarkierte Stammform (z.B. Hunde, Häuser, Autos) für den gesamten Plural oder als allgemeine Kasus-Numerusmarkierte Stammform (z.B. Menschen) für alle Kasus-Numerus-Kombinationen, ausgenommen den Nominativ Singular.

\begin{tabular}{|c|c|c|c|}
\hline \multicolumn{3}{|c|}{ I. STARK } & \multirow{2}{*}{$\begin{array}{c}\text { II. SCHWACH } \\
\text { Kasus-Numerus- } \\
\text { Stammformen }\end{array}$} \\
\hline & $\begin{array}{l}\text { Numerus- } \\
\text { Stammformen }\end{array}$ & & \\
\hline $\begin{array}{l}\text { A. NORMAL- } \\
\text { PLURAL }\end{array}$ & $\begin{array}{c}\text { B. VERSTÄRKTER } \\
\text { PLURAL }\end{array}$ & $\begin{array}{l}\text { C. SONDER- } \\
\text { PLURAL }\end{array}$ & \\
\hline $\begin{array}{c}-(e) / \mathrm{PL} \\
-(e) n / \mathrm{PL}_{\mathrm{F}}\end{array}$ & $\begin{array}{l}\mathrm{UL}_{-}(e) / \mathrm{PL} \\
\mathrm{UL}_{-e r} / \mathrm{PL}_{\mathrm{N}}\end{array}$ & $-s \# / P L$ & $-(e) n_{* \text { NOM.SG }}$ \\
\hline \multicolumn{3}{|c|}{ Kasusformen } & \\
\hline \multicolumn{3}{|c|}{$\begin{array}{c}-(e) s / \mathrm{GEN}_{* \mathrm{~F}, * \mathrm{PL}} \\
-(e) n / \mathrm{DAT}_{\mathrm{PL}}\end{array}$} & \\
\hline \multicolumn{4}{|l|}{ Beispiellexeme: } \\
\hline $\begin{array}{l}\text { HUND, M } \\
\text { SCHAF, N } \\
\text { KATZE, F }\end{array}$ & $\begin{array}{l}\text { BAUM, M } \\
\text { HAUS, N } \\
\text { HAND, F }\end{array}$ & $\begin{array}{l}\text { SNOB, M } \\
\text { AUTO, N } \\
\text { LOK, F }\end{array}$ & $\begin{array}{l}\text { MENSCH, M } \\
\text { AFFE, M }\end{array}$ \\
\hline $\begin{array}{l}\text { STRAHL, M|F } \\
\text { BETT, N|F } \\
\text { MÜHSAL, F|N }\end{array}$ & $\begin{array}{l}\text { GOTT, M } \mid \mathrm{N} \\
\text { FLOSS, } \mathrm{N} \mid \mathrm{M}\end{array}$ & & \\
\hline
\end{tabular}

M|F: Maskulinum mit femininer Pluralbildung, usw.

Tab. 7: Deutsch: Substantivflexive und Stammklassen

Die erste Einteilung der Substantive nach Stammklassen liefert die Klassen STARK und SCHWACH. Substantive mit einer Kasus-Numerus-markierten Stammform sind schwach, andere Substantive sind stark. Die starken Substantive bilden markierte Numerusstammformen, also Pluralstammformen. 
Nach den dabei verwendeten Suffixen sind drei Unterklassen zu unterscheiden: Substantive mit Normalplural, mit verstärktem Plural (oder Umlautplural) und mit Sonderplural (oder $s$-Plural). (Der leichteren Bezugnahme halber sind die Namen der Klassen in Tabelle 7 mit römischen Ziffern und Buchstaben versehen.) In Hinblick auf die Stammbildung müssen somit insgesamt vier Klassen von Substantiven unterschieden werden (IA, IB, IC, II). Ferner müssen Substantive mit ,unerwarteter' Pluralbildung (wie z.B. STRAHL, M F) als solche lexikalisch gekennzeichnet werden.

Neben den Stammformen können Substantive bis zu zwei reguläre Kasusformen (Formen, die auf bestimmte Einzelkasus festgelegt sind) besitzen. Wie die Tabelle verdeutlicht, wird aber in Hinblick auf die Kasusformenbildung keine zusätzliche Klassenbildung (über die für die Stammformenbildung notwendigen Unterscheidungen hinaus) benötigt. Sind die Stammformen eines Substantivs und sein Genus gegeben, so steht damit bereits fest, ob und gegebenenfalls welche Kasusformen gebildet werden. Bei gegebenem Flexivinventar lassen sich unter Beachtung der relevanten morphophonologischen Regeln (wie der Schwa-Regel für Substantive) die vollständigen Paradigmen ableiten. ${ }^{18}$ Die Vielfalt der Paradigmen (oder Flexionsklassen), die die Grammatikschreibung immer wieder vor Probleme gestellt hat, erweist sich als epiphänomenal.

Die anfangs aufgeworfene Frage nach dem Status der Deklinationsklassen in der deutschen Substantivflexion kann nun erneut gestellt werden. Die STARK-SCHWACH-Unterscheidung bei den Substantivlexemen wird in Hinblick auf die Flexion, genauer auf die Stammbildung, getroffen. Die Zugehörigkeit zu den Klassen I und II ist zwar in vielen Fällen aufgrund von Bedeutungs- und Formmerkmalen vorhersagbar, aber nicht generell aufgrund unabhängig gegebener Merkmale herleitbar. Die betreffende Klassifikation der Substantive stellt also eine Deklinationsklassenunterscheidung dar. Auch die Zugehörigkeit zu den Subklassen der starken Substantive, die in Hinblick auf die Pluralstammbildung unterschieden werden müssen (IA, IB, IC), ist nicht generell aufgrund unabhängig gegebener Merkmale herleitbar und stellt insofern ebenso eine Deklinationsklassenunterscheidung dar.

Im Ergebnis lässt sich festhalten, dass man bei der Beschreibung der deutschen Substantivflexion nicht ohne Deklinationsklassen auskommt. Die anzunehmende Klassifikation der Substantive nach ihrer Stammformenbildung teilt aber kaum Züge, kanonischer' Deklinationsklassensysteme (Corbett 2009), wie sie etwa am Lateinischen abgelesen werden können.

$18 \quad$ Wurzel (1994, S. 39) stellt fest, dass die Flexion eines Substantivs im Deutschen bei gegebener Grundform und NOM.PL-Form (wobei für die schwachen Substantive $n$-Plural angenommen wird) im Allgemeinen ,prädiktabel“ ist, wenn man eine passend angesetzte Menge von Regeln (,Paradigmenstrukturbedingungen`) annimmt. Die hier vorgeschlagene Analyse kommt ohne einen solchen Regelapparat aus. 
Insbesondere fehlt es völlig an den für die kanonische Ausprägung von Deklinationsklassen charakteristischen konkurrierenden Sätzen von klassentypischen Flexiven, die sich für alle oder wenigstens eine größere Anzahl von Kasus-Numerus-Kombinationen unterscheiden (vgl. Tabelle 1 mit Tabelle 7). An ihre Stelle ist eine kleine Menge von Mustern der Stammformenbildung getreten.

Die Stammformenbildung stellt den eigentlichen Kern der Substantivflexion des Deutschen dar, und nur sie macht eine Klassifikation der Substantive in Hinblick auf ihre Flexion erforderlich. Da die Substantive neben der unmodifizierten Stammform aber höchstens eine abgeleitete Stammform haben können, erschöpft sich die Aufgabe einer Deklinationsklasseneinteilung hier tatsächlich darin, für jedes Substantiv eben diese eine abgeleitete Form festzulegen. Zu bestimmen ist in jedem Fall nur, ob ein Substantiv eine Kasus-Numerus-markierte Stammform besitzt (also schwach flektiert wird) oder andernfalls, wie seine Pluralstammform gebildet wird.

Im Vorhergehenden sollte deutlich geworden sein, wie weit die deutsche Substantivflexion typologisch von Flexionssystemen wie dem des Lateinischen entfernt ist, auf die das Wort-und-Paradigma-Modell zugeschnitten ist: Grammatische Beschreibungen, die dem traditionellen Verfahren unkritisch folgen, können nur ein sehr verzerrtes Bild der tatsächlichen Verhältnisse geben, das wenig oder nichts zur Erklärung des Formenbaus beiträgt. ${ }^{19}$

Das Flexionssystem des gegenwärtigen Deutschen steht am vorläufigen Ende eines langen Prozesses, der im Ergebnis durch massive Vereinfachungen und Reduktionen geprägt ist. Schwankungsfälle bei der (Nicht-)Setzung von Kasusflexiven im Gegenwartsdeutschen mögen auf weiterhin zu erwartende Vereinfachungen hindeuten. Insgesamt lässt sich sagen, dass das Deutsche von einem Stadium, bei dem die Flexion der Substantive weitgehend oder überhaupt auf die Pluralbildung reduziert ist (wie im Englischen bzw. Italienischen), nicht allzu weit entfernt ist.

19 Vgl. die konsequent durchgeführte Beschreibung der nhd. Substantivflexion auf der Basis von Flexivsätzen (oder ,Relationsmorphemsätzen') in Simmler (1998, S. 195-228), die „die primäre Grundlage für die Festlegung der Substantivklassen“ (S. 216) darstellen sollen. Simmler geht von 13 Flexivsätzen mit 8 Elementen aus (z.B. $\{\varnothing$, , s, ə, $\varnothing$; ə, ə, ən, ə\} für Substantive wie FISCH), also 104 Systempositionen für Flexive, kommt aber mit weiteren Subunterscheidungen auf ca. 70 Paradigmen. Da die Flexivsätze als elementare, nicht weiter herleitbare Grundbausteine behandelt werden, kann das Modell nichts darüber sagen, wie die Flexivsätze zustande kommen und ebenso wenig darüber, wie sich die kleine Zahl von Flexiven auf die 104 Positionen verteilt, und insbesondere nichts zur Verteilung der mit Null belegten Positionen oder zu den vielfältigen Synkretismen. 


\section{Literatur}

Abondolo, Daniel (2009): Hungarian. In: Comrie, Bernard (Hg.): The world's major languages. 2. Aufl. London/New York, S. 484-496.

Acquaviva, Paolo (2008): Lexical plurals. A morphosemantic approach. (= Oxford Studies in Theoretical Linguistics 19). Oxford u.a.

Antonsen, Elmer H. (1973): Zur schwachen „Flexion“ im Deutschen. In: Linguistische Studien III. Festgabe für Paul Grebe zum 65. Geburtstag. Teil 1. (= Sprache der Gegenwart 23). Düsseldorf, S. 137-144.

Augst, Gerhard (1979): Neuere Forschungen zur Substantivflexion (Forschungsbericht). In: Zeitschrift für germanistische Linguistik 7, S. 220-232.

Bech, Gunnar (1963): Zur Morphologie der deutschen Substantive. In: Lingua 12, S. 177-189.

Bittner, Dagmar (1991): Von starken Feminina und schwachen Maskulina. Die neuhochdeutsche Substantivflexion - Eine Systemanalyse im Rahmen der natürlichen Morphologie. (= ZAS Papers in Linguistics 31). Diss. Jena. Internet: www.zas.gwzberlin.de/fileadmin/material/ZASPiL_Volltexte/zp31/zaspil31.pdf (Stand: 30.09.2011).

Bittner, Dagmar (1994): Die Bedeutung der Genusklassifikation für die Organisation der deutschen Substantivflexion. In: Köpcke (Hg.), S. 65-80.

Booij, Geert/Lehmann, Christian/Mugdan, Joachim (Hg.) (2000): Morphologie. Ein internationales Handbuch zur Flexion und Wortbildung. (= Handbücher zur Sprachund Kommunikationswissenschaft 17). Berlin u.a.

Bornschein, Matthias/Butt, Matthias (1987): Zum Status des s-Plurals im gegenwärtigen Deutsch. In: Abraham, Werner/Århammar, Ritva (Hg.): Linguistik in Deutschland. Akten des 21. Linguistischen Kolloquiums, Groningen 1986. (= Linguistische Arbeiten 182). Tübingen, S. 135-153.

Bräuer, Herbert (1969): Slavische Sprachwissenschaft. II: Formenlehre. 1. Teil. Berlin.

Carstairs, Andrew (1987): Allomorphy in inflexion. London/New York/Sydney.

Carstairs-McCarthy, Andrew (2000): Inflection classes. In: Booij/Lehmann/Mugdan (Hg.), S. 630-637.

Corbett, Greville G. (1982): Gender in Russian. An account of gender specification and its relationship to declension. In: Russian Linguistics 6, S. 197-232.

Corbett, Greville G. (2009): Canonical inflectional classes. In: Montermini, Fabio/Boyé, Gilles/Tseng, Jesse (Hg.): Selected Proceedings of the 6th Décembrettes: Morphology in Bordeaux. Somerville, MA, S. 1-11.

Curme, George O. (1922): A grammar of the German language. 2., überarb. Aufl. [9. Druck 1964]. New York.

D’Achille, Paolo/Thornton, Anna M. (2003): La flessione del nome dall'italiano antico all'italiano contemporaneo. In: Maraschio, Nicoletta/Poggi Salani, Teresa (Hg.): Italia linguistica anno Mille - Italia linguistica anno Duemila. (= Pubblicazioni della Società di Linguistica Italiana 45). Rom, S. 211-230. 
Dardano, Maurizio/Trifone, Pietro (1995): Grammatica italiana (con nozioni di linguistica). Bologna.

Duden-Grammatik (2009): Der Duden. Bd. 4: Die Grammatik. 8., überarb. Aufl. Hrsg. v.d. Dudenredaktion. Mannheim/Wien/Zürich.

Eisenberg, Peter (1998): Grundriß der deutschen Grammatik. Bd. 1: Das Wort. Stuttgart u.a.

Elekfi, László (1994): Magyar ragozási szótár. Dictionary of Hungarian inflections. Budapest.

Fleischer, Wolfgang (1974): Wortbildung der deutschen Gegenwartssprache. 3., überarb. Aufl. Leipzig.

Fourquet, Jean (1952): Grammaire de l'allemand. Paris.

Gallmann, Peter (1998): Case underspecification in morphology, syntax and the lexicon. In: Alexiadou, Artemis/Wilder, Chris (Hg.): Possessors, predicates and movement in the determiner phrase. (= Linguistik aktuell 22). Amsterdam/Philadelphia, S. $141-175$.

Gergely, György/Pléh, Csaba (1994): Lexical processing in an agglutinative language and the organization of the lexicon. In: Folia Linguistica 28, S. 175-204.

Göksel, Aslı/Kerslake, Celia (2005): Turkish. A comprehensive grammar. London/New York.

Grimm, Jacob (1837): Deutsche Grammatik. 4. Bd. Göttingen.

Halle, Morris/Marantz, Alec (2006): Clarifying ,Blur': paradigms, defaults and inflectional classes. In: Bachrach, Asaf/Nevins, Andrew (Hg.): Inflectional identity. (= Oxford Studies in Theoretical Linguistics 18). Oxford, S. 55-72.

Hegedűs, Rita (2006): PONS Substantivtabellen Ungarisch: übersichtlich und umfassend. Stuttgart u.a.

Köpcke, Klaus-Michael (1993): Schemata bei der Pluralbildung im Deutschen. Versuch einer kognitiven Morphologie. (= Studien zur deutschen Grammatik 47). Tübingen.

Köpcke, Klaus-Michael (Hg.) (1994): Funktionale Untersuchungen zur deutschen Nominal- und Verbalmorphologie. (= Linguistische Arbeiten 319). Tübingen.

Kornai, András (1992): Frequency in morphology. In: Kenesei, István (Hg.): Approaches to Hungarian. Bd. 4. Szeged, S. 246-268.

Kürschner, Sebastian (2008): Deklinationsklassen-Wandel. (= Studia linguistica Germanica 92). Berlin/New York.

Lehmann, Christian/Moravcsik, Edith (2000): Noun. In: Booij/Lehmann/Mugdan (Hg.), S. $732-757$.

Leumann, Manu (1977): Lateinische Laut- und Formenlehre. Neuausgabe der 19261928 erschienenen 5. Aufl. München.

Marcus, Gary F. et al. (1995): German inflection: the exception that proves the rule. In: Cognitive Psychology 29, S. 189-256. 
Müller, Gereon (2002): Remarks on nominal inflection in German. In: Kaufmann, Ingrid/Stiebels, Barbara (Hg.): More than words. A Festschrift for Dieter Wunderlich. (= Studia grammatica 53). Berlin, S. 113-145.

Neef, Martin (1998): The reduced syllable plural in German. In: Fabri, Ray/Ortmann, Albert/Parodi, Teresa (Hg.): Models of inflection. (= Linguistische Arbeiten 388). Tübingen, S. 244-265.

Nübling, Damaris (2008): Was tun mit Flexionsklassen? Deklinationsklassen und ihr Wandel im Deutschen und seinen Dialekten. In: Zeitschrift für Dialektologie und Linguistik 75, 3, S. 282-330.

Orzechowska, Alicja (1999): Paradygmatika. Rzeczownik [Paradigmatik. Nomen]. In: Grzegorczykowa, Renata/Laskowski, Roman/Wróbel, Henryk (Hg.): Gramatyka współczesnego Języka Polskiego [Grammatik der polnischen Gegenwartssprache]. Morfologia [1]. 3., revid. Aufl. Warschau, S. 270-332.

Paul, Hermann (1917): Deutsche Grammatik. Bd. II. Teil III: Flexionslehre. Halle a.d.S.

Paul, Hermann (1989): Mittelhochdeutsche Grammatik. 23. Aufl., neu bearb. v. Peter Wiehl und Siegfried Grosse. (= Sammlung kurzer Grammatiken germanischer Dialekte: A, Hauptreihe 2). Tübingen.

Plank, Frans (1991): Of abundance and scantiness in inflection: A typological prelude. In: Plank, Frans (Hg.): Paradigms. The economy of inflection. (= Empirical Approaches to Language Typology 9). Berlin/New York, S. 1-39.

Repetti, Lori (2006): The emergence of marked structures in the integration of loans in Italian. In: Gess, Randall S./Arteaga, Deborah L. (Hg.): Historical Romance linguistics: retrospective and perspectives. (= Studies in the Theory and History of Linguistic Science: Series 4, Current Issues in Linguistic Theory 274). Amsterdam/ Philadelphia, S. 209-235.

Simmler, Franz (1998): Morphologie des Deutschen. Flexions- und Wortbildungsmorphologie. (= Germanistische Lehrbuchsammlung 4). Berlin.

Steche, Theodor (1927): Die neuhochdeutsche Wortbiegung unter besonderer Berücksichtigung der Sprachentwicklung im 19. Jahrhundert. Erster Teil: Die Wortklassen - Die Beugung. Breslau.

Swan, Oscar E. (2002): A grammar of contemporary Polish. Bloomington.

Thieroff, Rolf (2000): Morphosyntax nominaler Einheiten im Deutschen. Habil.-Schrift. Bonn.

Thieroff, Rolf (2009): Über den Pluralumlaut beim Substantiv. In: Eins, Wieland/Schmöe, Friederike (Hg.): Wie wir sprechen und schreiben. Festschrift für Helmut Glück zum 60. Geburtstag. Wiesbaden, S. 29-44.

Törkenczy, Miklós (2002): Practical Hungarian grammar. Budapest.

Trubetzkoy, N. [=Trubeckoj, Nikolaj S.] (1934): Das morphonologische System der russischen Sprache. (= Description phonologique du russe moderne, partie 2; Travaux du Cercle linguistique de Prague 5,2). Prag/Leipzig. 
Vennemann, Theo (1982): Zur Silbenstruktur der deutschen Standardsprache. In: Vennemann, Theo (Hg.): Silben, Segmente, Akzente. (= Linguistische Arbeiten 126). Tübingen, S. 261-305.

Wegener, Heide (1995): Die Nominalflexion des Deutschen - verstanden als Lerngegenstand. (= Reihe Germanistische Linguistik 151). Tübingen.

Wiese, Bernd (1992): Unterspezifizierte Paradigmen. Form und Funktion in der pronominalen Deklination. Vortrag beim Symposium Funktionale Untersuchungen zur deutschen Nominal- und Verbalmorphologie am FAS, Berlin 22.5.1992. In: Linguistik online 4, 3/1999. Internet: www.linguistik-online.de/3_99/wiese.html (Stand: 05.06.2011).

Wiese, Bernd (2000): Warum Flexionsklassen? Über die deutsche Substantivdeklination. In: Thieroff, Rolf et al. (Hg.): Deutsche Grammatik in Theorie und Praxis. Tübingen, S. 139-153.

Wiese, Bernd (2004): Categories and paradigms. On underspecification in Russian declension. In: Müller, Gereon/Gunkel, Lutz/Zifonun, Gisela (Hg.): Explorations in nominal inflection. (= Interface Explorations 10). Berlin/New York, S. 321-372.

Wiese, Bernd (2009): Variation in der Flexionsmorphologie: Starke und schwache Adjektivflexion nach Pronominaladjektiven. In: Konopka, Marek/Strecker, Bruno (Hg.): Deutsche Grammatik - Regeln, Normen, Sprachgebrauch. (= Jahrbuch des Instituts für Deutsche Sprache 2008). Berlin/New York, S. 166-194.

Wiese, Bernd (2011): Optimal specifications: On case marking in Polish. In: Nolda, Andreas/Teuber, Oliver (Hg.): Syntax and Morphology Multidimensional. (= Interface Explorations 24). Berlin/Boston, S. 101-127.

Wiese, Bernd (i.Ersch.): On Latin nominal inflection: the form-function relationship. In: Morphology.

Wunderlich, Dieter (1999): German noun plural reconsidered. In: Behavioral and Brain Sciences 22, S. 1044-1045.

Wurzel, Wolfgang U. (1984): Flexionsmorphologie und Natürlichkeit. Ein Beitrag zur morphologischen Theoriebildung. (= Studia grammatica 21). Berlin.

Wurzel, Wolfgang U. (1994): Gibt es im Deutschen noch eine einheitliche Substantivflexion? Oder: Auf welche Weise ist die deutsche Substantivflexion möglichst angemessen zu erfassen? In: Köpcke (Hg.), S. 29-44. 